IMA Journal of Numerical Analysis (2013) Page 1 of 18 doi:10.1093/imanum/drn000

\title{
GMRES convergence bounds that depend on the right-hand side vector
}

\author{
David Titley-Peloquin $\dagger$, Jennifer Pestana $\ddagger$, And Andrew J. Wathen \\ Mathematical Institute, University of Oxford, Oxford, OX1 3LB, UK
}

[Received on 13 April 2013]

\begin{abstract}
We consider the convergence of the algorithm GMRES of Saad and Schultz for solving linear equations $B x=b$, where $B \in \mathbb{C}^{n \times n}$ is nonsingular and diagonalizable, and $b \in \mathbb{C}^{n}$. Our analysis explicitly includes the initial residual vector $r_{0}$. We show that the GMRES residual norm satisfies a weighted polynomial least-squares problem on the spectrum of $B$, and that GMRES convergence reduces to an ideal GMRES problem on a rank-one modification of the diagonal matrix of eigenvalues of $B$. Numerical experiments show that the new bounds can accurately describe GMRES convergence.
\end{abstract}

Keywords: GMRES; convergence analysis; iterative methods; linear systems; Krylov subspace methods

\section{Introduction}

Let $x_{k}$ denote the $k$-th iterate of the algorithm GMRES (Saad \& Schultz, 1986) applied to $B x=b$, $B \in \mathbb{C}^{n \times n}, b \in \mathbb{C}^{n}$ with corresponding residual vector $r_{k} \equiv b-B x_{k}$. By definition,

$$
\left\|r_{k}\right\|_{2}=\min _{\substack{q \in \Pi_{k} \\ q(0)=1}}\left\|q(B) r_{0}\right\|_{2}
$$

where $\Pi_{k}$ denotes the set of polynomials of degree at most $k$. For a more thorough description of the algorithm and its implementation details, see Saad \& Schultz (1986) or, for example, the textbooks Greenbaum (1997) or Saad (1996).

Obtaining generally descriptive convergence bounds for GMRES has been an active research topic since the algorithm was introduced in Saad \& Schultz (1986) and is still to date considered a difficult open problem. A typical first step in deriving convergence bounds is the following, which is an immediate consequence of (1.1):

$$
\frac{\left\|r_{k}\right\|_{2}}{\left\|r_{0}\right\|_{2}} \leqslant \min _{\substack{q \in \Pi_{k} \\ q(0)=1}}\|q(B)\|_{2} .
$$

The polynomial approximation problem in (1.2) is widely referred to as the ideal GMRES problem (Greenbaum \& Trefethen, 1994). Bounds on (1.2) can be obtained using the spectral decomposition of $B$ (if $B$ is diagonalizable) (Saad \& Schultz, 1986), its field of values (Beckermann et al., 2006; Eiermann, 1993; Eiermann \& Ernst, 2001; Eisenstat et al., 1983; Liesen \& Tichý, 2012), or its pseudospectra (Trefethen, 1990). An overview of these approaches is given in Simoncini \& Szyld (2007, §6).

\footnotetext{
${ }^{\dagger}$ Email:David.Titley-Peloquin@maths.ox.ac.uk. The work of this author was supported by an NSERC of Canada PDF Fellowship.

${ }^{\ddagger}$ Corresponding author. Email: Jennifer.Pestana@maths.ox.ac.uk

${ }^{\S}$ Email: Andy.Wathen@maths.ox.ac.uk
}

(c) The author 2013. Published by Oxford University Press on behalf of the Institute of Mathematics and its Applications. All rights reserved. 
The bound (1.2) is a worst-case bound that holds over all $r_{0} \in \mathbb{C}^{n}$. In practice, however, one has a specific $B$ and a specific $r_{0}$, most likely not the worst-case. Consequently, (1.2) may not be descriptive of actual GMRES convergence. In short, practical convergence bounds should take into account the effect of the initial residual vector $r_{0}$. Indeed, $r_{0}$ determines the Krylov subspace from which the approximation to $x$ is sought. Arguments along these general lines have also been made, for instance, in Toh (1997), Driscoll et al. (1998, §8), Liesen \& Strakoš $(2004, \S 3.1)$, Liesen \& Strakoš $(2010, \S 3)$, and Duintjer Tebbens \& Meurant (2012). Some analyses that explicitly include $r_{0}$ can be found in Ipsen (2000) (when $B$ is a Jordan block), Liesen \& Strakoš (2004) and Li \& Zhang (2009) (when $B$ is Toeplitz tridiagonal), and for more general matrices $B$ in Liesen (2000). In this note, by considering the influence of the right-hand side vector, we attempt to further identify situations in which convergence is much faster than would be predicted by (1.2).

In Section 2 we present convergence bounds based on weighted polynomial least-squares problems on the spectrum of $B$. In Section 3 we show that the convergence of GMRES applied to $B x=b$ reduces to the ideal GMRES problem applied to a rank-one modification of the diagonal matrix of eigenvalues of $B$ and provide some analysis of this rank-one modification. Numerical experiments are presented in Section 4 that demonstrate the performance of these bounds. Throughout this paper we assume that $B$ is nonsingular and diagonalizable, and that GMRES is implemented in exact arithmetic.

\section{Weighted polynomial approximation problems on the spectrum}

Suppose that $B$ is diagonalizable, i.e. $B=Z \Lambda Z^{-1}$ with $\Lambda$ diagonal. Then from (1.2),

$$
\frac{\left\|r_{k}\right\|_{2}}{\left\|r_{0}\right\|_{2}} \leqslant \kappa_{2}(Z) \min _{\substack{q \in \Pi_{k} \\ q(0)=1}} \max _{\lambda \in \sigma(B)}|q(\lambda)|,
$$

where $\kappa_{2}(Z) \equiv\|Z\|_{2}\left\|Z^{-1}\right\|_{2}$ and $\sigma(B)$ is the spectrum of $B$. If $\kappa_{2}(Z)$ is not much larger than 1 , the above upper bound is governed by the eigenvalues of $B$. This, of course, is generally not the case for highly non-normal matrices, for which $\kappa_{2}(Z) \gg 1$.

It is well-known that eigenvalues alone do not generally determine the convergence of GMRES. Indeed, Greenbaum, Pták, \& Strakoš (1996) have shown that for any given set of $n$ eigenvalues and any given non-increasing convergence curve, there exists a matrix $B$ with the given eigenvalues and a vector $b$ such that GMRES applied to $B x=b$ starting with $x_{0}=0$ produces the given convergence curve. Furthermore, Arioli, Pták, \& Strakoš (1998) have given parametrizations of the set of all matrix-vector pairs $\{B, b\}$ such that GMRES applied to $B x=b$ starting with $x_{0}=0$ produces the prescribed curve. Matrices $B$ in this set can be chosen to have any eigenvalues. Recently, Duintjer Tebbens \& Meurant (2012) showed that it is possible to additionally prescribe the Ritz values at every iteration.

We summarize the Arioli et al. result for diagonalizable matrices with distinct eigenvalues below (but note that the result holds for any nonderogatory matrix):

Theorem 2.1 (Arioli, Pták, \& Strakoš (1998)). For any given $v_{i} \in \mathbb{R}$ satisfying

$$
v_{0} \geqslant v_{1} \geqslant \cdots \geqslant v_{n-1}>0, \quad v_{n}=0,
$$

and distinct nonzero $\lambda_{i} \in \mathbb{C}, i=1, \ldots, n$, the following statements are equivalent:

1. The spectrum of $B$ is $\left\{\lambda_{i}\right\}$, and GMRES applied to $B x=b$ starting from $x_{0}=0$ produces residual vectors $r_{k}$ such that $\left\|r_{k}\right\|_{2}=v_{k}$. 
2. The matrix $B$ has the form $B=U Y C Y^{-1} U^{*}$ and $b=U h$, where $U$ is unitary,

$$
Y=\left[\begin{array}{cc}
h & R \\
\eta_{n} & 0
\end{array}\right], \quad h=\left[\eta_{1}, \ldots, \eta_{n-1}\right]^{T}, \quad \eta_{k}=\sqrt{v_{k-1}^{2}-v_{k}^{2}},
$$

$R$ is nonsingular and upper-triangular, and $C$ is the companion matrix of the monic polynomial $\prod_{i=1}^{n}\left(\lambda-\lambda_{i}\right)$.

It is clear from the above that the prescribed convergence curve is closely related to the right-hand side vector $b$, and the interaction between the eigenvectors of $B$ with $b$. This highlights the importance of $b$ (more generally, if $x_{0} \neq 0$, of $r_{0}$ ) in determining the convergence of GMRES. For instance, if $B$ has distinct eigenvalues, the companion matrix $C$ is diagonalizable as

$$
C=V^{-1} \Lambda V, \quad V=\left[\begin{array}{ccc}
\lambda_{1} & \ldots & \lambda_{1}^{n} \\
\vdots & & \vdots \\
\lambda_{n} & \ldots & \lambda_{n}^{n}
\end{array}\right]
$$

In this case, the following statement is equivalent to the second in Theorem 2.1: $B$ has a diagonalization $B=Z \Lambda Z^{-1}$ with $Z=U Y V^{-1}$, and $b=U h$. In other words, the parametrization reveals which structure the matrix of eigenvectors and $b$ must have in order to obtain a given convergence curve starting with $x_{0}=0$ from a matrix with prescribed distinct eigenvalues.

Motivated by the above, we attempt to derive bounds on $\left\|r_{k}\right\|_{2} /\left\|r_{0}\right\|_{2}$ that involve the interplay between $Z$ and $r_{0}$. As a simple example, note that from (1.1) we can write

$$
\frac{\left\|r_{k}\right\|_{2}}{\left\|r_{0}\right\|_{2}} \leqslant\|Z\|_{2} \frac{\left\|Z^{-1} r_{0}\right\|_{2}}{\left\|r_{0}\right\|_{2}} \min _{\substack{q \in \Pi_{k} \\ q(0)=1}} \max _{\lambda \in \sigma(B)}|q(\lambda)|
$$

where

$$
\frac{1}{\|Z\|_{2}} \leqslant \frac{\left\|Z^{-1} r_{0}\right\|_{2}}{\left\|r_{0}\right\|_{2}} \leqslant\left\|Z^{-1}\right\|_{2}
$$

If $\left\|Z^{-1} r_{0}\right\|_{2} /\left\|r_{0}\right\|_{2}$ is close to its lower bound in (2.4), then the right-hand side of (2.3) is determined almost solely by the eigenvalues of $B$. In this case, when $\kappa_{2}(Z) \gg 1$, the bound (1.2) can, and (2.1) certainly does, fail to be descriptive of actual GMRES convergence. The following theorem further characterizes the effect of the vector $Z^{-1} r_{0}$ and of eigenvalues on GMRES convergence.

THEOREM 2.2 Suppose that $B$ has the diagonalization $B=Z \Lambda Z^{-1}$ with $\Lambda=\operatorname{diag}\left(\lambda_{i}\right)$, and let $w=$ $Z^{-1} r_{0} /\left\|r_{0}\right\|_{2}=W e$, where $W=\operatorname{diag}\left(w_{i}\right)$ and $e=[1, \ldots, 1]^{T}$. The GMRES residuals in (1.1) satisfy

$$
\frac{\left\|r_{k}\right\|_{2}}{\left\|r_{0}\right\|_{2}} \leqslant\|Z\|_{2} \min _{\substack{q \in \Pi_{k} \\ q(0)=1}}\|W q(\Lambda) e\|_{2}=\|Z\|_{2} \min _{\substack{q \in \Pi_{k} \\ q(0)=1}}\left(\sum_{i=1}^{n}\left|w_{i}\right|^{2}\left|q\left(\lambda_{i}\right)\right|^{2}\right)^{1 / 2}
$$

Proof. For any polynomial $q$,

$$
q(B) \frac{r_{0}}{\left\|r_{0}\right\|_{2}}=Z q(\Lambda) w=Z q(\Lambda) W e=Z W q(\Lambda) e
$$


From this and the definition (1.1) we have

$$
\frac{\left\|r_{k}\right\|_{2}}{\left\|r_{0}\right\|_{2}}=\min _{\substack{q \in \Pi_{k} \\ q(0)=1}}\|Z W q(\Lambda) e\|_{2} \leqslant\|Z\|_{2} \min _{\substack{q \in \Pi_{k} \\ q(0)=1}}\|W q(\Lambda) e\|_{2}
$$

giving (2.5).

Theorem 2.2 shows that the GMRES relative residual is bounded above by $\|Z\|_{2}$ times the residual of a polynomial least-squares approximation problem on the spectrum of $B$, weighted by the diagonal matrix $W=\operatorname{diag}\left(w_{i}\right)$. Related ideas have previously been used to describe the convergence of GMRES and the conjugate gradient method (see, for example, Axelsson \& Kaporin, 2000; Jennings, 1977; van der Vorst \& Vuik, 1993). The bound (2.5) can be rewritten as a weighted linear least-squares problem

$$
\min _{\substack{q \in \Pi_{k} \\ q(0)=1}}\|W q(\Lambda) e\|_{2}=\min _{t \in \mathbb{C}^{k}}\left\|W\left(e+V_{k} t\right)\right\|_{2}
$$

where $V_{k} \in \mathbb{C}^{n \times k}$ consists of the first $k$ columns of the Vandermonde matrix $V$ in (2.2). Alternatively, we can think of (2.5) in terms of GMRES applied to $\Lambda \widetilde{x}=w$, since

$$
\min _{\substack{q \in \Pi_{k} \\ q(0)=1}}\|W q(\Lambda) e\|_{2}=\min _{\substack{q \in \Pi_{k} \\ q(0)=1}}\|q(\Lambda) w\|_{2}
$$

is the residual vector computed when GMRES is used to solve $\Lambda \widetilde{x}=w$, provided that the initial iterate is the zero vector. We use this fact in our numerical experiments in Section 4.

In (2.5), the influence of the non-normality of $B$ on convergence is fully contained in $\|Z\|_{2}$ and the diagonal matrix of weights $W$. (Note that we can always scale the columns of $Z$ such that $\|Z\|_{2}=1$. Of course, the scaling also modifies the matrix of weights $W$.) If $B$ is normal, (2.5) is in fact an equality in which $\|Z\|_{2}=1$ and $\sum_{i=1}^{n}\left|w_{i}\right|^{2}=1$. On the other hand, if $B$ is non-normal, the $\left|w_{i}\right|$ may be very large.

Note that the upper bounds (2.3) and (2.5) coincide at $k=0$, but (2.5) is never larger than (2.3) since

$$
\min _{\substack{q \in \Pi_{k} \\ q(0)=1}}\left(\sum_{i=1}^{n}\left|w_{i}\right|^{2}\left|q\left(\lambda_{i}\right)\right|^{2}\right)^{1 / 2} \leqslant\|w\|_{2} \min _{\substack{q \in \Pi_{k} \\ q(0)=1}} \max _{i}\left|q\left(\lambda_{i}\right)\right|=\frac{\left\|Z^{-1} r_{0}\right\|_{2}}{\left\|r_{0}\right\|_{2}} \min _{\substack{q \in \Pi_{k} \\ q(0)=1}} \max _{\substack{ \\q(B)}}|q(\lambda)| .
$$

In other words, the bounds (1.2), (2.1) and (2.3) ignore the weights $w_{i}$ in their respective polynomial approximation problems. In some situations, insight into convergence can be gained by explicitly considering these weights.

THEOREM 2.3 In the notation of Theorem 2.2, for any subset of indices $\mathscr{J}$ with $|\mathscr{J}|=p$, the GMRES residuals in (1.1) with $k>p$ satisfy

$$
\frac{\left\|r_{k}\right\|_{2}}{\left\|r_{0}\right\|_{2}} \leqslant\|Z\|_{2} \min _{\substack{q \in \Pi_{k-p} \\ q(0)=1}}\left(\sum_{\substack{i=1 \\ i \notin \mathcal{J}}}^{n}\left|\tilde{w}_{i}\right|^{2}\left|q\left(\lambda_{i}\right)\right|^{2}\right)^{1 / 2} \leqslant\|Z\|_{2}\|\tilde{w}\|_{2} \min _{\substack{q \in \Pi_{k-p} \\ q(0)=1}} \max _{\substack{q \in \sigma(B) \\ q(B)}}|q(\lambda)|,
$$

where

$$
\tilde{w}_{i}=w_{i} \prod_{j \in \mathscr{J}}\left(1-\frac{\lambda_{i}}{\lambda_{j}}\right), \quad \tilde{w}=\left[\tilde{w}_{i}\right] \in C^{n}
$$


Proof. With the above-defined $\tilde{w}_{i}$, for $k>p$ let

$$
\tilde{q}_{k}(\lambda)=q_{k-p}(\lambda) \prod_{j \in \mathscr{J}}\left(1-\frac{\lambda}{\lambda_{j}}\right), \quad q_{k-p}(\lambda)=\arg \min _{\substack{q \in \Pi_{k-p} \\ q(0)=1}}\left(\sum_{\substack{i \neq 1 \\ i \notin \mathcal{J}}}^{n}\left|\tilde{w}_{i}\right|^{2}\left|q\left(\lambda_{i}\right)\right|^{2}\right)^{1 / 2} .
$$

Clearly $\tilde{q}_{k} \in \Pi_{k}$ and $\tilde{q}_{k}(0)=1$. Substituting $\tilde{q}_{k}$ into (2.5) and defining $\widetilde{W}=\operatorname{diag}\left(\tilde{w}_{i}\right)$ we obtain

$$
\frac{\left\|r_{k}\right\|_{2}}{\left\|r_{0}\right\|_{2}} \leqslant\|Z\|_{2}\left\|W \tilde{q}_{k}(\Lambda) e\right\|_{2}=\|Z\|_{2}\left\|\widetilde{W} q_{k-p}(\Lambda) e\right\|_{2}=\|Z\|_{2} \min _{\substack{q \in \Pi_{k-p} \\ q(0)=1}}\left(\sum_{\substack{i=1 \\ i \notin \mathcal{J}}}^{n}\left|\tilde{w}_{i}\right|^{2}\left|q\left(\lambda_{i}\right)\right|^{2}\right)^{1 / 2}
$$

Note that, in the above theorem, $i \in \mathscr{J}$ implies $\tilde{w}_{i}=0$. In other words, the polynomial $\tilde{q}_{k}$ eliminates the effect of both the eigenvalues $\lambda_{i}$ as well as the non-normality associated with indices $i \in \mathscr{J}$.

Of course, (2.6) is an upper bound on (2.5) since

$$
\min _{\substack{q \in \Pi_{k} \\ q(0)=1}}\|W q(\Lambda) e\|_{2} \leqslant\left\|W \tilde{q}_{k}(\Lambda) e\right\|_{2}=\min _{\substack{q \in \Pi_{k-p} \\ q(0)=1}}\left(\sum_{i=1}^{n}\left|\tilde{w}_{i}\right|^{2}\left|q\left(\lambda_{i}\right)\right|^{2}\right)^{1 / 2}
$$

The bound (2.6) bears resemblance to (4.1) in Campbell et al. (1996), in which the eigenvalues of $B$ are separated into two sets, one containing eigenvalues that lie in a cluster and the other comprising the $p$ remaining outliers. The outliers do not contribute to the convergence rate in the Campbell et al. model after the $p$-th iteration (but do contribute to the asymptotic error constant). The bound in Theorem 2.3 is more general, since any eigenvalues, not just outliers, can form the set $\left\{\lambda_{i}\right\}_{i \in \mathscr{J}}$. Moreover, the nonnormality associated with large weights $w_{i}, i \in \mathscr{J}$, does not appear in our bound at all, since $\tilde{w}_{i}=0$ whenever $i \in \mathscr{J}$.

Of course, (2.6) is not too helpful if many/all components of $w$ are large. Nevertheless, it shows that even (2.3) can sometimes be overly pessimistic provided $w$ has only a few large entries corresponding to large in magnitude or clustered eigenvalues.

A concrete example is given in Example 4.1 in Section 4. The same example also appears in Huhtanen \& Nevanlinna (2000), where lower bounds on $\left\|r_{k}\right\|_{2} /\left\|r_{0}\right\|_{2}$ are given based on the fact that $B$ is a rank-one perturbation of a normal matrix. However, the distance in rank from a normal matrix alone cannot fully explain the observed convergence, as we shall demonstrate in the next section.

\section{Reduction to ideal GMRES of a diagonal plus rank-one matrix}

The following bounds are simple to derive but may help to give some additional insight into the convergence of GMRES when $B$ is highly non-normal.

LEMMA 3.1 Suppose that $B$ has the diagonalization $B=Z \Lambda Z^{-1}$. For any nonsingular $K \in \mathbb{C}^{n \times n}$, the 
GMRES residuals in (1.1) satisfy

$$
\begin{aligned}
\frac{\left\|r_{k}\right\|_{2}}{\left\|r_{0}\right\|_{2}} & \leqslant\|K\|_{2}\left\|K^{-1} \tilde{r}\right\|_{2} \min _{\substack{q \in \Pi_{k} \\
q(0)=1}}\left\|q\left(K^{-1} B K\right)\right\|_{2} \\
& \leqslant\|K\|_{2}\left\|K^{-1} \tilde{r}\right\|_{2} \kappa_{2}\left(K^{-1} Z\right) \min _{\substack{q \in \Pi_{k} \\
q(0)=1}} \lambda \in \sigma(B) \\
\max & |q(\lambda)|,
\end{aligned}
$$

where $\tilde{r}=r_{0} /\left\|r_{0}\right\|_{2}$.

Proof. From (1.1),

$$
\begin{aligned}
\frac{\left\|r_{k}\right\|_{2}}{\left\|r_{0}\right\|_{2}} & =\min _{\substack{q \in \Pi_{k} \\
q(0)=1}}\left\|K K^{-1} q(B) K K^{-1} r_{0}\right\|_{2} /\left\|r_{0}\right\|_{2} \\
& \leqslant\|K\|_{2}\left\|K^{-1} \tilde{r}\right\|_{2} \min _{\substack{q \in \Pi_{k} \\
q(0)=1}}\left\|q\left(K^{-1} B K\right)\right\|_{2} .
\end{aligned}
$$

Using the diagonalization $B=Z \Lambda Z^{-1}$ in the above we obtain

$$
\frac{\left\|r_{k}\right\|_{2}}{\left\|r_{0}\right\|_{2}} \leqslant\|K\|_{2}\left\|K^{-1} \tilde{r}\right\|_{2} \kappa_{2}\left(K^{-1} Z\right) \min _{\substack{q \in \Pi_{k} \\ q(0)=1}} \max _{\lambda \in \sigma(B)}|q(\lambda)| .
$$

If $K$ is any unitary matrix, the bound (3.1) reduces to the ideal GMRES problem (1.2). In (3.2), any unitary $K$ leads to the standard bound (2.1) while the choice $K=Z$ gives (2.3).

It is easy to verify that if $K$ is chosen such that $\tilde{r} \in \mathscr{U}$, where $\mathscr{U}$ denotes the span of the left singular vectors corresponding to the largest singular value of $K$, then $\left\|K^{-1} \tilde{r}\right\|_{2}=1 /\|K\|_{2}$. From Lemma 3.1 we then immediately obtain

$$
\frac{\left\|r_{k}\right\|_{2}}{\left\|r_{0}\right\|_{2}} \leqslant \min _{\substack{q \in \Pi_{k} \\ q(0)=1}}\left\|q\left(K^{-1} B K\right)\right\|_{2} \leqslant \kappa_{2}\left(K^{-1} Z\right) \min _{\substack{q \in \Pi_{k} \\ q(0)=1}} \max _{\lambda \in \sigma(B)}|q(\lambda)| .
$$

The above may help to understand why the standard bounds (1.2) and (2.1) sometimes severely overestimate $\left\|r_{k}\right\|_{2} /\left\|r_{0}\right\|_{2}$. For instance, in (2.1) any column scaling can be applied to $Z$ in order to minimize its condition number. (Equilibrating the columns is a near-optimal strategy, as shown by van der Sluis (1969).) The bound in (3.3) shows that we have much more freedom: $Z$ can also be scaled on the left with any nonsingular matrix $K$, the only restriction being that $\tilde{r} \in \mathscr{U}$.

Unfortunately, we have not found a simple way to pick $K$ with $\tilde{r} \in \mathscr{U}$ to minimize $\left\|q\left(K^{-1} B K\right)\right\|_{2}$ or $\kappa_{2}\left(K^{-1} Z\right)$. However, it is still possible to choose $K$ with $\tilde{r} \notin \mathscr{U}$ so that $\left\|K^{-1} \tilde{r}\right\|_{2} \ll\left\|K^{-1}\right\|_{2}$ and $\|K\|_{2}\left\|K^{-1} \tilde{r}\right\|_{2}$ in (3.1) is small. The following theorem identifies one such choice.

THEOREM 3.1 Suppose that $B \in \mathbb{C}^{n \times n}$ has a diagonalization $B=Z \Lambda Z^{-1}$ with $\Lambda=\operatorname{diag}\left(\lambda_{i}\right)$. Define

$$
v_{j} \equiv\left(\Lambda-\lambda_{j} I\right) w, \quad w=Z^{-1} r_{0} /\left\|r_{0}\right\|_{2}=Z^{-1} \tilde{r} .
$$

Then for any index $j \in[1, n]$ such that $w_{j}=e_{j}^{T} w \neq 0$, with $e_{j}$ the $j$-th unit vector in $\mathbb{C}^{n}$, the GMRES residuals in (1.1) satisfy

$$
\frac{\left\|r_{k}\right\|_{2}}{\left\|r_{0}\right\|_{2}} \leqslant\left(1+\|Z\|_{2}\right) \min _{\substack{q \in \Pi_{k} \\ q(0)=1}}\left\|q\left(\Lambda+v_{j} e_{j}^{T}\right)\right\|_{2}
$$


Proof. Pick $K$ in Lemma 3.1 to be the matrix $Z$ with its $j$-th column replaced by $\tilde{r}=r_{0} /\left\|r_{0}\right\|_{2}$, i.e.,

$$
K=Z+\left(\tilde{r}-Z e_{j}\right) e_{j}^{T}
$$

Under the assumption that $w_{j}=e_{j}^{T} Z^{-1} \tilde{r} \neq 0$, this chosen $K$ is nonsingular. Additionally,

$$
K e_{j}=Z e_{j}+\tilde{r}\left(e_{j}^{T} e_{j}\right)-Z e_{j}\left(e_{j}^{T} e_{j}\right)=\tilde{r}
$$

and

$$
\begin{aligned}
& \|K\|_{2} \leqslant\|Z\|_{2}\left\|I-e_{j} e_{j}^{T}\right\|_{2}+\|\tilde{r}\|_{2} \leqslant 1+\|Z\|_{2}, \\
& \left\|K^{-1} \tilde{r}\right\|_{2}=\left\|K^{-1} K e_{j}\right\|_{2}=1,
\end{aligned}
$$

where we have used (3.6) to obtain the latter result. Thus with the above choice of $K$ the term $\|K\|_{2}\left\|K^{-1} \tilde{r}\right\|_{2}$ in (3.1) is not large, provided $\|Z\|_{2}$ is not large, even if $K$ (and/or $Z$ ) is very ill-conditioned. Furthermore, with $w=Z^{-1} \tilde{r}$,

$$
Z^{-1} K=I+\left(w-e_{j}\right) e_{j}^{T}
$$

and using the Sherman-Morrison identity (see, e.g., Higham (2002, page 487), or Golub \& Van Loan (1996, page 50) for the more general Sherman-Morrison-Woodbury identity) we have

$$
K^{-1} Z=I-\frac{1}{w_{j}}\left(w-e_{j}\right) e_{j}^{T}
$$

where $w_{j}=e_{j}^{T} w$. Thus,

$$
\begin{aligned}
K^{-1} B K=K^{-1} Z \Lambda Z^{-1} K & =\left[\Lambda-\frac{\lambda_{j}}{w_{j}}\left(w-e_{j}\right) e_{j}^{T}\right]\left[I+\left(w-e_{j}\right) e_{j}^{T}\right] \\
& =\Lambda+\left[\Lambda-\frac{\lambda_{j}}{w_{j}} I-\frac{\lambda_{j} e_{j}^{T}\left(w-e_{j}\right)}{w_{j}} I\right]\left(w-e_{j}\right) e_{j}^{T} \\
& =\Lambda+\left[\Lambda-\frac{1}{w_{j}}\left(\lambda_{j}+\lambda_{j} w_{j}-\lambda_{j}\right) I\right]\left(w-e_{j}\right) e_{j}^{T} \\
& =\Lambda+\left(\Lambda-\lambda_{j} I\right) w e_{j}^{T} \\
& =\Lambda+v_{j} e_{j}^{T} .
\end{aligned}
$$

Substituting this along with (3.7) into (3.1) completes the proof.

Theorem 3.1 shows that the convergence of GMRES applied to $B x=b$ is essentially determined by $\|Z\|_{2}$ along with the ideal GMRES problem on the matrix $\Lambda+v_{j} e_{j}^{T}$, a rank-one modification of the diagonal matrix of eigenvalues of $B$. (As in the previous section, we can scale the columns of $Z$ so that $\|Z\|_{2}=1$, which of course modifies $v_{j}$. With this scaling the right-hand side of (3.5) is no larger than two.) The rank-one modification $v_{j} e_{j}^{T}$ depends on both the eigenvalues and eigenvectors of $B$, as well as on the right-hand side vector. It depends additionally on $j$, the index that determines which eigenvector is replaced in $Z$ to form $K$, and it is certainly possible to choose a different $j$ at each iteration $k$ or to obtain a number of bounds for a number of columns $j$. In this way, (3.5) is akin to pseudospectral bounds, where a parameter can also be varied. The bound (3.5) is also similar to the bound in Huhtanen (1999), where GMRES convergence bounds for $B x=b$ are obtained by considering the convergence of 
GMRES applied to systems of the form $\left(B+b v^{*}\right) y=b$, for arbitrary $v \in \mathbb{C}^{n}$. In contrast to (3.5) above, however, Huhtanen's bound includes the condition number of the eigenvector matrix of this perturbed system.

There has been some work on the convergence of ideal GMRES applied to the sum of a normal and a low-rank matrix (see, e.g., Nevanlinna, 1996; Huhtanen \& Nevanlinna, 2000). Theorem 3.1 can be viewed as a negative result in this direction. If an arbitrary rank-one modification $v_{j} e_{j}^{T}$ of the diagonal matrix $\Lambda$ had little effect on ideal GMRES, then from (3.5) with the scaling $\|Z\|_{2}=1$ we would have

$$
\frac{\left\|r_{k}\right\|_{2}}{\left\|r_{0}\right\|_{2}} \leqslant 2 \min _{\substack{q \in \Pi_{k} \\ q(0)=1}}\left\|q\left(\Lambda+v_{j} e_{j}^{T}\right)\right\|_{2} \approx 2 \min _{\substack{q \in \Pi_{k} \\ q(0)=1}}\|q(\Lambda)\|_{2}=2 \min _{\substack{q \in \Pi_{k} \\ q(0)=1}} \max _{\lambda \in \sigma(B)}|q(\lambda)|,
$$

i.e., convergence of GMRES applied to $B x=b$ would generally be determined by the eigenvalues of $B$. As discussed in the introduction, this is certainly not the case.

Nevertheless, there may be specific systems and specific $v_{j}$ in (3.4) for which the quantities

$$
\min _{\substack{q \in \prod_{k} \\ q(0)=1}}\left\|q\left(\Lambda+v_{j} e_{j}^{T}\right)\right\|_{2}
$$

and

$$
\min _{\substack{q \in \Pi_{k} \\ q(0)=1}}\|q(\Lambda)\|_{2}=\min _{\substack{q \in \Pi_{k} \\ q(0)=1}} \max _{\lambda \in \sigma(B)}|q(\lambda)|
$$

do not differ too much.

Let us examine the difference between $\left\|q\left(\Lambda+v_{j} e_{j}^{T}\right)\right\|_{2}$ and $\|q(\Lambda)\|_{2}$. Since $e_{j}^{T} v_{j}=0$,

$$
\left(\Lambda+v_{j} e_{j}^{T}\right)^{i}=\Lambda^{i}+\left(\sum_{r=0}^{i-1} \lambda_{j}^{r} \Lambda^{i-1-r}\right) v_{j} e_{j}^{T}
$$

and so, for any polynomial

$$
q(\lambda)=1+\sum_{i=1}^{k} \alpha_{i} \lambda^{i}
$$

we have

$$
q\left(\Lambda+v_{j} e_{j}^{T}\right)=q(\Lambda)+\sum_{i=1}^{k} \alpha_{i}\left(\sum_{r=0}^{i-1} \lambda_{j}^{r} \Lambda^{i-1-r}\right) v_{j} e_{j}^{T}
$$

Therefore,

$$
\left\|q\left(\Lambda+v_{j} e_{j}^{T}\right)-q(\Lambda)\right\|_{2}=\left\|\sum_{i=1}^{k} \sum_{r=0}^{i-1} \alpha_{i} \lambda_{j}^{r} \Lambda^{i-1-r} v_{j}\right\|_{2}=\left\|\Theta_{j} w\right\|_{2},
$$

where $\Theta_{j}=\left(\sum_{i=1}^{k} \sum_{r=0}^{i-1} \alpha_{i} \lambda_{j}^{r} \Lambda^{i-1-r}\right)\left(\Lambda-\lambda_{j} I\right)$ is a singular diagonal matrix. Thus, ensuring that $\left\|q\left(\Lambda+v_{j} e_{j}^{T}\right)-q(\Lambda)\right\|_{2}$ is small is reduced to a diagonal scaling $\Theta_{j}$ for $w$, where $\Theta_{j}$ depends on the coefficients $\alpha_{i}$ of the polynomial $q(z)$. In the context of GMRES, $q(z)$ is an ideal GMRES polynomial. Unfortunately, even for a diagonal matrix the ideal GMRES polynomial is difficult to determine. It can in principle be computed by semidefinite programming (see, for example, Toh \& Trefethen, 1998); however, in our experience, it is not possible to solve the resulting optimization problem accurately for large values of $k$. Thus, it seems difficult to compute $\alpha_{i}, i=1, \ldots, k$, and the optimal $\Theta_{j}$, in general. 
We additionally find that the bound (3.5) can never be significantly worse than (2.3). By applying (3.8) and (3.9) we find that

$$
q\left(\Lambda+v_{j} e_{j}^{T}\right)=K^{-1} Z q(\Lambda) Z^{-1} K=q(\Lambda) Z^{-1} K-\left(w-e_{j}\right) e_{j}^{T} q(\Lambda)=q(\Lambda)+q(\Lambda) w e_{j}^{T}-w e_{j}^{T} q(\Lambda)
$$

from which it follows that

$$
\frac{\left\|r_{k}\right\|_{2}}{\left\|r_{0}\right\|_{2}} \leqslant\left(1+\|Z\|_{2}\right)\left(1+2\|w\|_{2}\right) \min _{\substack{q \in \Pi_{k} \\ q(0)=1}}\|q(\Lambda)\|_{2} .
$$

If $Z$ is scaled so that $\|Z\|_{2}=1$, the constant becomes $2+4\|w\|_{2}$. We are interested in systems for which $\|w\|_{2} \gg 1$, in which case (3.5) is no more than a factor approximately four times larger than (2.3). In our numerical experiments, we find that (3.5) is typically much smaller than (2.3) and is never larger.

As for a comparison between our bounds (2.5) and (3.5): clearly, when $k=0,(2.5)$ can be much larger than (3.5). For general $k$, however, we have not found a simple relationship between the two bounds. In our numerical experiments, (2.5) is often much larger, particularly at early iterations, but it can also be much smaller than (3.5).

We have introduced a new bound (3.5) which shows that the relative residuals of GMRES are bounded by the constant factor $1+\|Z\|_{2}$ and an ideal GMRES problem on a rank-one perturbation of $\Lambda$, the matrix of eigenvalues of $B$. In the next section we present numerical experiments that demonstrate how well this bound, and (2.5), describe GMRES convergence.

\section{Numerical experiments}

In this section we illustrate the performance of the bounds (2.5), (2.6) and (3.5) and compare them with (1.2), (2.1) and (2.3). All computations were performed using MATLAB with ideal GMRES polynomials computed using the igmres function in the SDPT3 toolbox (Toh et al., 1999). In all experiments the initial guess is $x_{0}=0$ and the termination criterion is that $\left\|r_{k}\right\|_{2} /\left\|r_{0}\right\|_{2}<10^{-10}$. Excluding the first example, we scale the eigenvector matrix $Z$ to have unit spectral norm, so that the constant factor $1+\|Z\|_{2}$ in (3.5) is equal to two.

As discussed in Section 2, the bound (2.5) is equivalent to the residual norm obtained when GMRES is applied to $\Lambda \widetilde{x}=w$ with a zero initial vector. Consequently, we use these GMRES residuals to compute (2.5) in our numerical experiments. To compute (3.5) we select at each iteration $k$ the column index $j$ (and vector $v_{j}$ ) for which (3.5) is smallest.

EXAMPLE 4.1 The behaviour of GMRES in the this first example was left as an open question in Greenbaum \& Strakoš (1994). Let $B=Z D Z^{-1}$ and $b=[1, \ldots, 1]$, where

$$
Z=\left[\begin{array}{ccccc}
1 & \sqrt{1-\delta} & 0 & \ldots & 0 \\
0 & \sqrt{\delta} & 0 & \ldots & 0 \\
0 & 0 & 1 & \ldots & 0 \\
\vdots & \vdots & \vdots & \ddots & \vdots \\
0 & 0 & 0 & \ldots & 1
\end{array}\right], \quad D=\left[\begin{array}{ccccc}
20 & & & & \\
& 10 & & & \\
& & \mu_{3} & & \\
& & & \ddots & \\
& & & & \mu_{n}
\end{array}\right]
$$

with $\mu_{i}, i=3, \ldots, n$ samples of a uniformly distributed random variable in $(1,5)$. We choose $\delta=$ $1 \times 10^{-12}$ and $n=20$. For this problem $\|Z\|_{2}=\sqrt{2}$. 

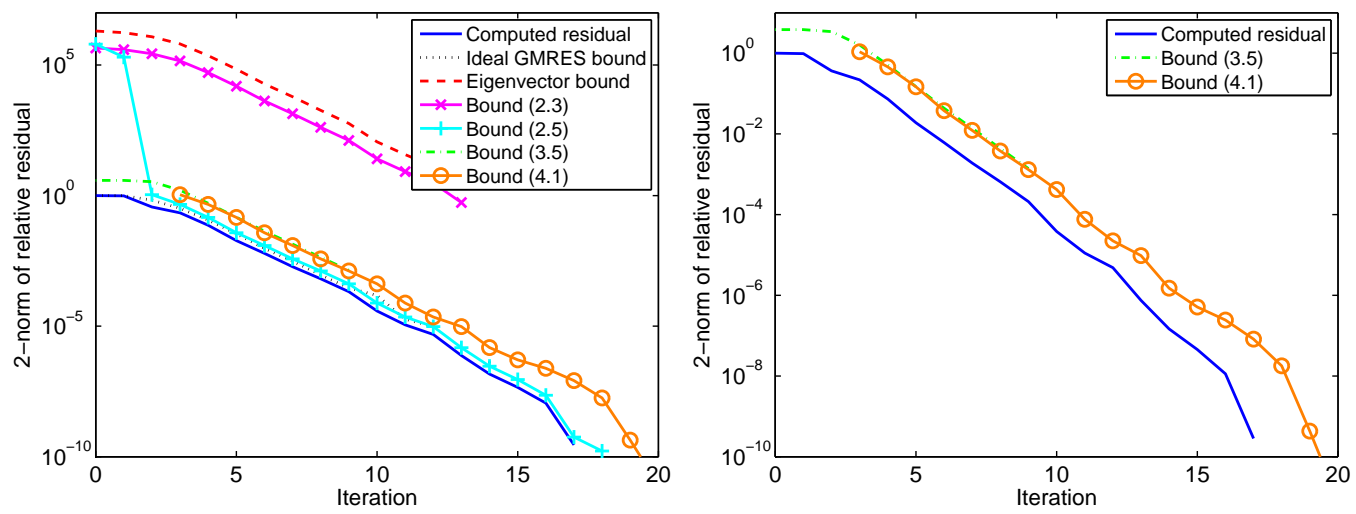

FIG. 1: Left: the bounds (1.2), (2.1), (2.3), (2.5) and (4.1) and computed relative residuals for the problem in Example 4.1. Right: the bounds (3.5) and (4.1) and relative residuals with a different scale.

Theorem 2.3 clearly shows why the non-normality of $B$ has little effect on convergence in this case. Note that all the eigenvalues of $B$ are normal, except for the two large well-separated eigenvalues $\lambda_{1}$ and $\lambda_{2}$. (See e.g. Horn \& Johnson, 1999, $\S 1.6 .5$ for the definition of normal eigenvalues.) Consequently, for any initial residual $r_{0}, \sum_{i=3}^{n}\left|w_{i}\right|^{2} \leqslant 1$. With $\mathscr{J}=\{1,2\}$, we have

$$
\left|\tilde{w}_{i}\right| \leqslant\left|w_{i}\right|\left(1-\frac{1}{20}\right)\left(1-\frac{1}{10}\right) \leqslant\left|w_{i}\right|, \quad i>2,
$$

so that $\|\tilde{w}\|_{2} \leqslant 1$ and from (2.6), for $k>2$,

$$
\begin{aligned}
\frac{\left\|r_{k}\right\|_{2}}{\left\|r_{0}\right\|_{2}} & \leqslant \sqrt{2} \min _{\substack{q \in \Pi_{k-2} \\
q(0)=1}}\left(\sum_{i=3}^{n}\left|\tilde{w}_{i}\right|^{2}\left|q\left(\lambda_{i}\right)\right|^{2}\right)^{1 / 2} \\
& \leqslant \sqrt{2} \min _{\substack{q \in \Pi_{k-2} \\
q(0)=1}} \max _{\substack{ \\
q(B)}}|q(\lambda)| .
\end{aligned}
$$

Thus, after two iterations, an upper bound independent of the non-normality of $B$ applies.

The relative GMRES residual norms for this problem were computed; we see from Figure 1 that GMRES stagnates for one iteration, in line with our prediction above, before exhibiting a fairly constant convergence rate.

Figure 1 also shows the bounds (1.2), (2.1), (2.3), (2.5) and (4.1). It is clear that, in addition to predicting the end of the stagnation phase, (4.1) accurately describes the convergence of GMRES from iteration $k=3$ onwards. Although the bound (2.5) is inaccurate at the first step, when it is heavily influenced by $\|w\|_{2}=4.5 \times 10^{5}$, from iteration $k=2$ it is also descriptive, suggesting that (2.5) quickly accounts for the non-normality of the eigenvalues of $B$ in this example.

For this matrix the ideal GMRES bound (1.2) is also descriptive. This is due to the fact that the $w_{i}$ satisfy $\sum_{i=3}^{n}\left|w_{i}\right|^{2} \leqslant 1$ for any $r_{0} \in \mathbb{C}^{n}$, as discussed above. There are certainly matrices for which this is not the case (see, for instance, Example 4.2). Neither (2.1) nor (2.3) is close to the GMRES convergence curve since the condition number of the eigenvectors and $\|w\|_{2}$ are both large. The failure 
of (2.1) and (2.3) to describe GMRES convergence shows the value of considering the weights $w_{i}$ (or $\left.\tilde{w}_{i}\right)$ in the polynomial approximation problem.

We also see from Figure 1 (c) that (3.5) lies close to the computed residuals. Although (2.5) is slightly closer to the computed GMRES residuals at later iterations, during the initial stagnation period (3.5) is much more descriptive because the intercept is orders of magnitude smaller. Note that (3.5) cannot be computed when $k$ is larger than nine because the ideal GMRES polynomials of $\Lambda+v_{j} e_{j}^{T}$ cannot be obtained from the igmres function in SDPT3 for any column index $j$ because of rounding errors.

EXAMPLE 4.2 Our next example is a finite difference discretization of the two-dimensional convectiondiffusion equation

$$
-v \nabla^{2} u+s \cdot \nabla u=0 \text { in } \Omega=(0,1) \times(0,1), \quad u=g \text { on } \partial \Omega
$$

with $s=[0,1]^{T}$ and boundary conditions

$$
g(x, 0)=x, g(0, y)=-1, g(1, y)=1, g(x, 1)=0 .
$$

This equation has a particular right-hand side and we might expect that bounds that incorporate this information will be more descriptive than those that do not.

Following Hemmingsson (1998) we let $n_{x}$ and $n_{y}$ be the number of grid points used in the discretization in the $x$ and $y$ directions, respectively, so that $x_{k}=k h_{x}, k=1, \ldots, n_{x}$, and $y_{j}=j h_{y}, j=1, \ldots n_{y}$, where

$$
h_{x}=\frac{1}{n_{x}+1} \text { and } h_{y}=\frac{1}{n_{y}+1} .
$$

The second-order centered finite difference approximation of (4.2) leads to equations of the form

$$
-v\left(\frac{u_{k+1, j}-2 u_{k, j}+u_{k-1, j}}{h_{x}^{2}}+\frac{u_{k, j+1}-2 u_{k, j}+u_{k, j-1}}{h_{y}^{2}}\right)+\frac{u_{k, j+1}-u_{k, j-1}}{2 h_{y}}=0,
$$

where $k=1, \ldots, n_{x}, j=1, \ldots, n_{y}$.

Let

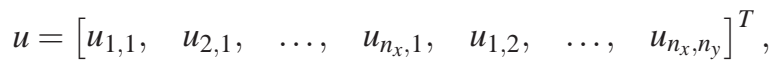

$$
\begin{aligned}
& \alpha_{x}=0, \quad \alpha_{y}=\frac{1}{h_{y}}, \quad \beta_{i}=\frac{2 v}{h_{i}^{2}}, \quad i=x, y,
\end{aligned}
$$

and

$$
B_{i}=\left[\begin{array}{cccc}
2 \beta_{i} & \alpha_{i}-\beta_{i} & & \\
-\alpha_{i}-\beta_{i} & \ddots & \ddots & \\
& \ddots & \ddots & \alpha_{i}-\beta_{i} \\
& & -\alpha_{i}-\beta_{i} & 2 \beta_{i}
\end{array}\right], \quad i=x, y .
$$

Then $u$ is the solution of $B x=b$, where $B=I_{n_{y}} \otimes B_{x}+B_{y} \otimes I_{n_{x}}$ and

$$
b=\left[\begin{array}{lllllll}
b_{1,1}, & b_{2,1}, & \ldots, & b_{n_{x}, 1}, & b_{1,2}, & \ldots, & b_{n_{y}, n_{x}}
\end{array}\right]^{T},
$$



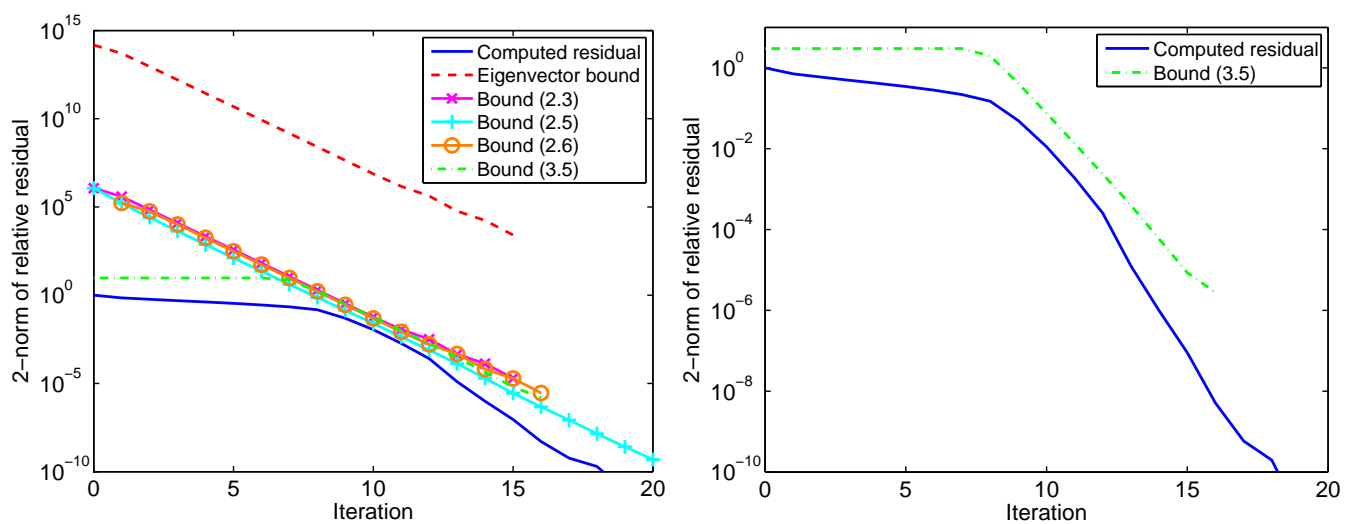

FIG. 2: Left: the bounds (1.2), (2.1), (2.3), (2.5) and (3.5) and computed relative residuals for the finite difference discretization of the convection-diffusion equation in Example 4.2. Right: the bound (3.5) and relative residuals with a different scale.

with

$$
b_{k, j}= \begin{cases}\frac{v}{h_{x}^{2}}, & k=0, n_{x}, j=1, \ldots, n_{y}, \\ 0, & \text { otherwise. }\end{cases}
$$

We choose $n_{x}=5$ and $n_{y}=18$, so that $B$ has dimension 90 , and let $v=0.0275$. We choose $\mathscr{J}=\left\{j_{\max }\right\}$ in (2.6), where $j_{\max }=67$ is the index of the largest element of $w$ in absolute value.

The behaviour of GMRES applied to this system is well known (see, e.g., Liesen \& Strakoš, 2004, 2005; Duintjer Tebbens et al., 2010); the relative residuals stagnate and then have a faster second phase of convergence. All the bounds capture the asymptotic convergence rate reasonably well. However, only (3.5) correctly captures the initial stagnation. In addition, because the intercept is small, the bound is close to the relative residuals.

The ideal GMRES polynomial also has these two phases of convergence. However, the stagnation phase is significantly longer than that of the computed residuals or (3.5). The bound (2.1) that involves the condition number of the eigenvector matrix, on the other hand, does not capture this two-phase behaviour. Instead, it decreases linearly and, because of the ill-conditioning of the eigenvectors of $B$ (the condition number computed by MATLAB is $1.5 \times 10^{14}$ ), it is orders of magnitude larger the relative residual curve. The bounds (2.3), (2.5) and (2.6) also decrease linearly and while the intercepts are smaller than that of (2.3), they are still relatively large since $\|w\|_{2}=1.6 \times 10^{6}$. Consequently, these bounds are not descriptive at early iterations, although they almost coincide with (3.5) during the second phase of convergence. We note that adding more indices to $\mathscr{J}$ does not change (1.1); since $w$ has many large components in magnitude, this is not surprising.

EXAMPLE 4.3 Let us now explore the performance of the bounds as the right-hand side vector of the problem in Example 4.2 varies. To effect this, we alter the boundary condition as in Example 2.2 in Liesen \& Strakoš (2005). Specifically, we let

$$
g(1, \eta)=1, \quad k h_{y} \leqslant \eta<1, \quad k=1, \ldots, n_{y},
$$



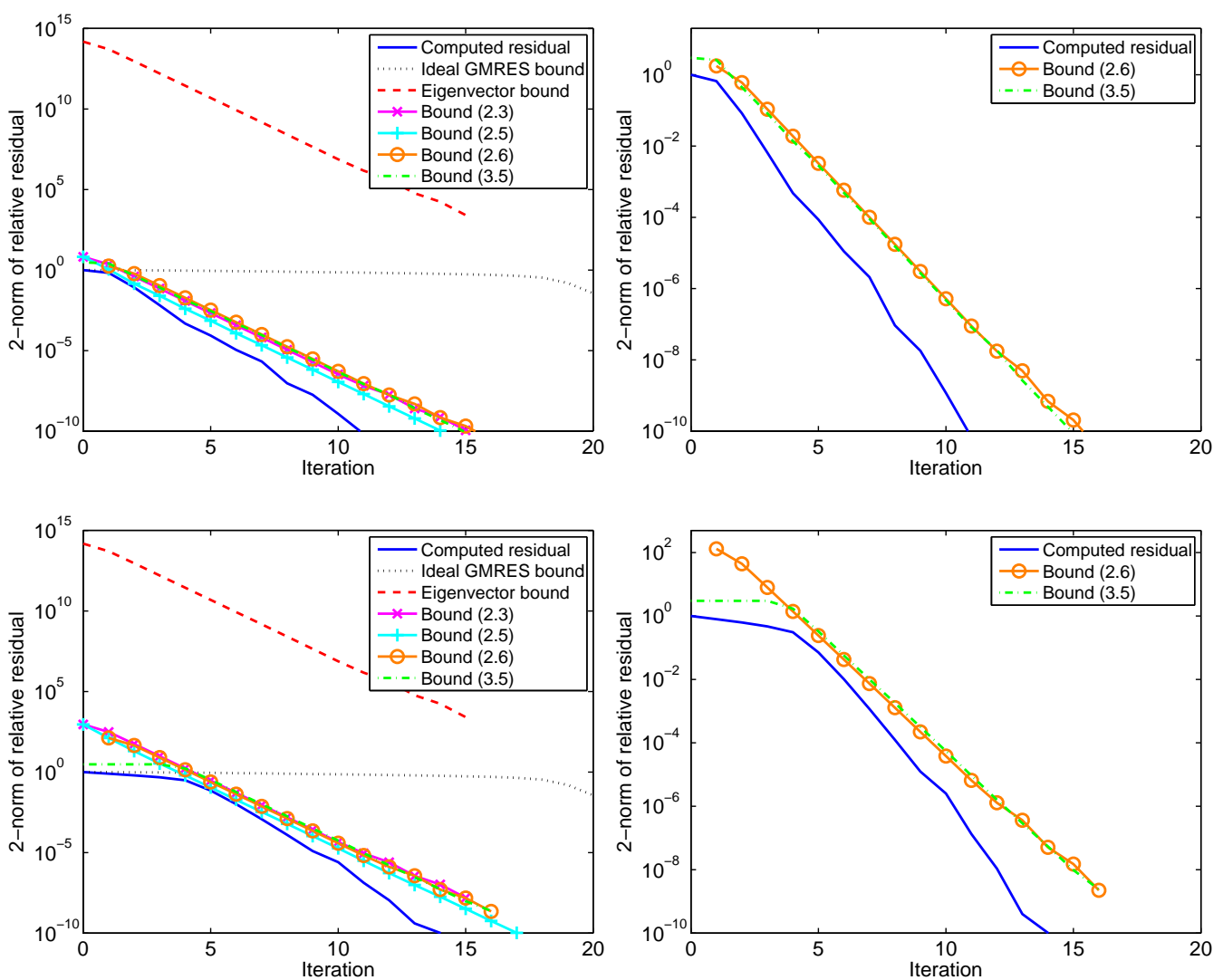

FIG. 3: Left: the bounds (1.2), (2.1), (2.3), (2.5) and (3.5) and computed relative residuals for the finite difference discretization of the convection-diffusion equation in Example 4.3 with $\eta=18$ (top) and $\eta=14$ (bottom). Right: the bound (3.5) and relative residuals with a different scale with $\eta=18$ (top) and $\eta=14$ (bottom).

with homogeneous Dirichlet conditions elsewhere on the boundary. The other parameters are unchanged from those in Example 4.2.

It is clear, from comparison of Figures 2 and 3, that the right-hand side has a dramatic impact on the convergence of GMRES for this problem. However, the bound (3.5) is able to capture the changing convergence behaviour. While (2.3), (2.5) and (2.6) fail to capture the initial stagnation they are certainly reasonable predictors of the second phase of convergence. However, the intercepts of (2.3), (2.5) and (2.6) must increase as the stagnation phase lengthens, to capture the second phase of convergence. We also find that (1.2) and (2.1), that do not depend on the right-hand side vector, are poor for all chosen right-hand side vectors.

EXAMPLE 4.4 We consider a right-preconditioned linear system arising from a discretized partial differential equation (PDE). Again, the resulting linear system has a specific right-hand side that should be 
considered when predicting GMRES convergence.

The specific problem is an SUPG finite element discretization of the convection-diffusion equation (Elman et al., 2005, Example 3.3)

$$
-v \nabla^{2} u+s \cdot \nabla u=0 \text { on }(-1,1) \times(-1,1), \quad u(x, y)= \begin{cases}1, & x=1 \\ 1, & y=-1 \text { and } x>0 \\ 0, & \text { elsewhere on the boundary }\end{cases}
$$

where $s=\left[-\sin \frac{\pi}{6}, \cos \frac{\pi}{6}\right]^{T}$ and $v=0.005$. We use IFISS (Silvester $e$ t al., 2011) with default settings to perform the discretization and obtain a matrix $B \in \mathbb{R}^{289 \times 289}$. Application of the geometric multigrid preconditioner in Ramage (1999), also computed by IFISS using default settings, gives rise to the rightpreconditioned system $B P^{-1} y=b, x=P^{-1} y$ to which we apply right-preconditioned GMRES. For this problem it was not possible to compute, using igmres, the ideal GMRES polynomials of $B$. We are also unable to compute (2.5) at later iterations because the in-built GMRES function in MATLAB terminated, having computed a backward stable solution to $\Lambda \tilde{x}=w$. When computing (2.6) we again choose $\mathscr{J}=\left\{j_{\max }\right\}$, where $j_{\max }=\arg \max _{i}\left|w_{i}\right|$.

It is clear from Figure 4 that the preconditioner is very effective, as the relative residuals decrease extremely rapidly. However, the standard bound (2.1) is not descriptive of true GMRES convergence for this example because $Z$, the matrix of eigenvectors of $B P^{-1}$, has 2-norm condition number $1.1 \times 10^{10}$. The bound (2.3) is slightly better but is still orders of magnitude larger than the computed relative residuals since $\|w\|_{2}=2.7 \times 10^{7}$. At $k=0,(2.5)$ must also equal the norm of $w$ but this bound quickly drops and is then descriptive. Additionally, (2.6) is descriptive, even though $\mathscr{J}$ contains only one index, so that only one element of $w$ is "removed".

The constant in (3.5) is two, as in previous examples, and (3.5) is a good predictor of convergence for this problem, as can be seen from Figure 4. Although $B P^{-1}$ is highly non-normal, this non-normality does not significantly affect the convergence of right-preconditioned GMRES applied to this particular problem, with this particular right-hand side vector. The bounds (2.5), (2.6) and (3.5) are able to capture the interaction between $B P^{-1}$ and $b$, which is missed by (2.1) and (2.3).

EXAMPLE 4.5 Finally, we examine the arc130 and fs_183_3 matrices from the University of Florida Sparse Matrix Collection (Davis \& Hu, 2011), right-preconditioned by the ILU(0) preconditioner (Meijerink \& van der Vorst, 1977). We set $x=[1, \ldots, 1]^{T}$. For neither problem can the ideal GMRES polynomial of $B$ be computed using igmres.

The matrix arc130 has dimension 130. Figure 5 shows that, similarly to Example 4.4, the relative GMRES residuals for arc130 decrease extremely rapidly. Since the condition number of $Z$ is $4.3 \times 10^{15}$ and $\|w\|_{2}=1.3 \times 10^{10}$, both (2.1) and (2.3) are orders of magnitude larger than the computed relative residuals. As in previous examples, (2.5) is initially large because of the size of $\|w\|_{2}$ but is then the most descriptive bound. Although $Z$ is ill-conditioned, the intercept of (3.5) is small, and so this bound is rather close to the computed residuals at all iterations, in contrast to (2.1) and (2.3). Again, in (2.6) we let $j_{\max }=110$ bet the index of the largest element of $w$ in magnitude and set $\mathscr{J}=\left\{j_{\max }\right\}$ in (2.6); doing so makes the bound reasonably descriptive.

GMRES also converges fairly quickly for the $183 \times 183$ matrix fs_183_3. For this example, the computed matrix of eigenvectors $Z$ is numerically singular, with $\kappa_{2}(Z) \approx 6.4 \times 10^{17}$, while the norm of $w$ computed by MATLAB is $8.9 \times 10^{14}$. It is, therefore, unsurprising that neither (2.1) nor (2.3) is close to the computed relative residuals. The large norm of $w$ also makes the computation of the bound (2.5) 

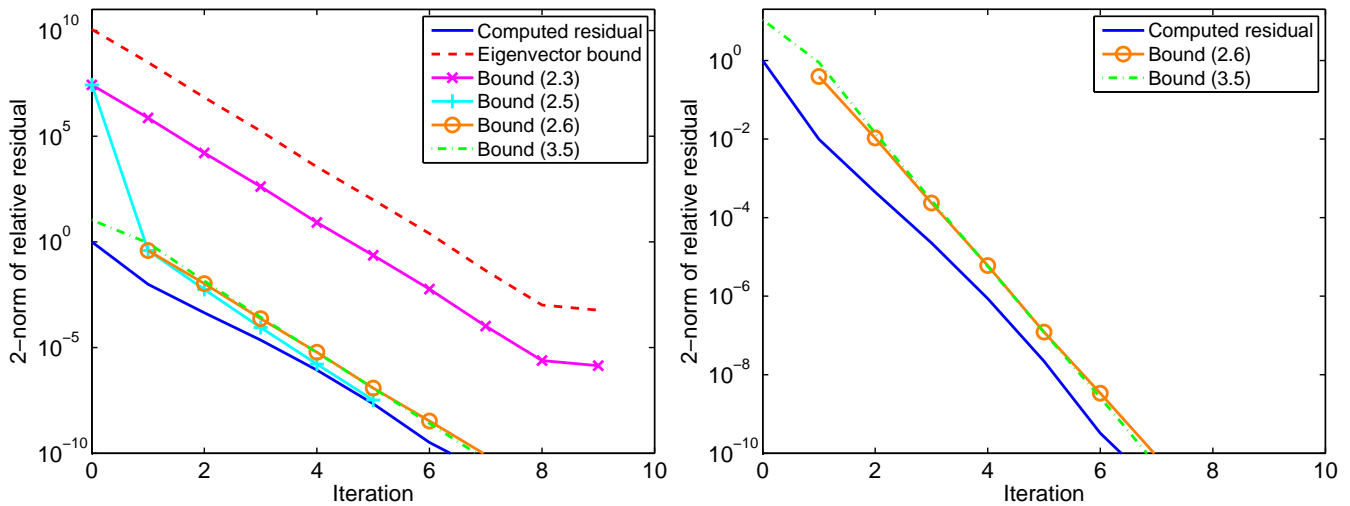

FIG. 4: Left: the bounds (2.1), (2.3), (2.5), (2.6) and (3.5) and computed relative residuals for the convection-diffusion problem in Example 4.4. Right: the bounds (2.6) and (3.5) and relative residuals with a different scale.

difficult, although we do see that it drops dramatically after one iteration and is then close to (3.5). For this example, $\min _{j}\left\|v_{j}\right\|=837$, which is certainly not small. However, the bound (3.5) is, as in the previous examples, an excellent predictor of convergence. We compute (2.6), including in $\mathscr{J}$ the indices corresponding to the $p$ largest values of $w$ in absolute value, with $p=1,2$ and 9. From Figure 6 we find that (2.6) is not so descriptive when $p=1$, while with $p=2,(2.6)$ is initially similar to (3.5) but then stagnates. This stagnation at later iterations is due to the ideal GMRES polynomial, computed by igmres, which does not reduce in norm. We remark that there is little difference between (2.6) with $p=2$ and $p=3,4,5$, indicating that while removal of the two largest components affects (2.6) significantly, several more components must be removed before the intercept of the bound decreases further. When $p=9$, the bound (2.6) does differ, but is not as descriptive as (3.5).

\section{Conclusions}

We have introduced bounds for GMRES applied to diagonalizable matrices which take into account the starting residual vector $r_{0}$. In particular, Theorem 2.2 show that convergence of GMRES applied to $B x=b$ reduces to a weighted least-squares problem on the spectrum of $B$, while Theorem 3.1 shows that convergence of GMRES applied to $B x=b$ reduces to the ideal GMRES problem applied to $\Lambda+v_{j} e_{j}^{T}$, a rank-one modification of the diagonal matrix of eigenvalues of $B$. Our numerical experiments show that these bounds can accurately describe GMRES convergence in cases where the standard bound (2.1) fails to be descriptive. Thus, we feel that these bounds are a worthwhile contribution towards a better understanding of the convergence of the algorithm. 

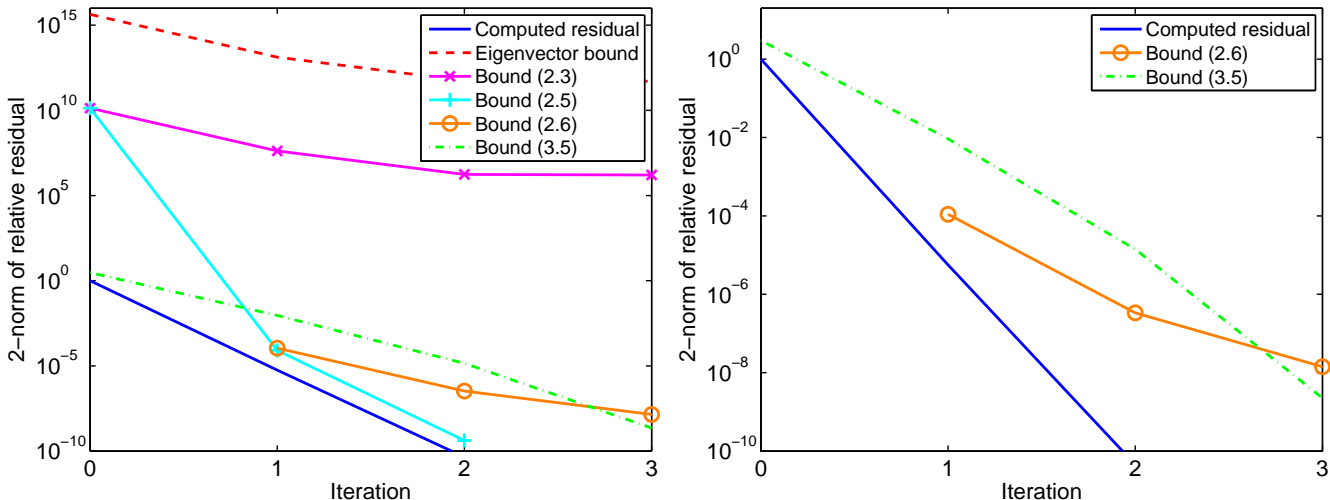

FIG. 5: Left: the bounds (2.1), (2.3), (2.5) and (3.5) and computed relative residuals for arc130 in Example 4.5. Right: the bound (3.5) and relative residuals with a different scale.
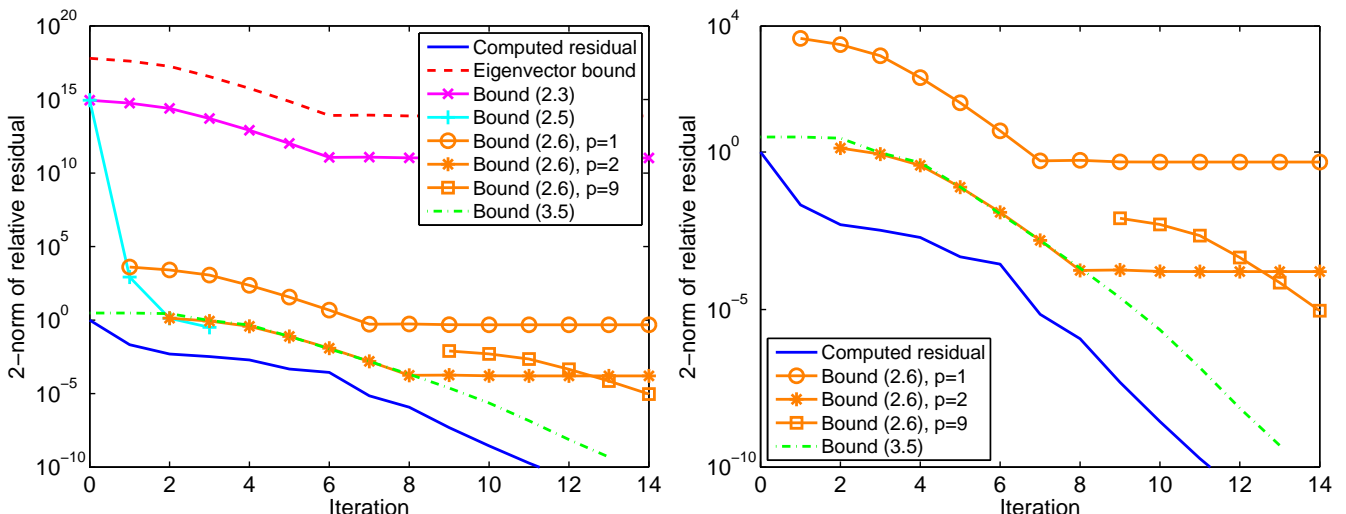

FIG. 6: Left: the bounds (2.1), (2.3), (2.5) and (3.5) and computed relative residuals for fs_183_3 in Example 4.5. Right: the bound (3.5) and relative residuals with a different scale. 


\section{Acknowledgements}

We thank the referees for their helpful comments and suggestions that have improved the manuscript. The first author would like to thank Jörg Liesen for helpful discussions and for pointing out the references Huhtanen \& Nevanlinna (2000) and Nevanlinna (1996), and Miroslav Rozložník and Zdeněk Strakoš for helpful discussions. The second author would like to thank Kim-Chuan Toh for advice on using the SDPT3 package.

\section{REFERENCES}

Arioli, M., Pták, V. \& Strakoš, Z. (1998) Krylov sequences of maximal length and convergence of GMRES. BIT, 38, 636-643.

AXELSSON, O. \& KAPORIN, I. (2000) On the sublinear and superlinear rate of convergence of conjugate gradient methods. Numer. Algorithms, 25, 1-22.

Beckermann, B., Goreinov, S. A. \& Tyrtyshnikov, E. E. (2006) Some remarks on the Elman estimate for GMRES. SIAM J. Matrix Anal. Appl., 27, 772-778.

CAmpbell, S. L., IPSen, I. C. F., Kelley, C. T. \& Meyer, C. D. (1996) GMRES and the minimal polynomial. BIT, 36, 664-675.

Davis, T. A. \& Hu, Y. (2011) The University of Florida Sparse Matrix Collection. ACM T. Math. Software, 38, $1-25$.

Driscoll, T. A., Toh, K.-C. \& Trefethen, L. N. (1998) From potential theory to matrix iterations in six steps. SIAM Rev., 40, 547-578.

Duintjer Tebbens, J., Liesen, J. \& Strakoš, Z. (2010) Analysis of the second phase of the GMRES convergence for a convection-diffusion model problem. Technical Report. Prague, Czech Republic: Faculty of Mathematics and Physics, Charles University of Prague.

Duintjer Tebbens, J. \& Meurant, G. (2012) Any Ritz value behavior is possible for Arnoldi and for GMRES. SIAM J. Matrix Anal. Appl., 33, 958-978.

Eiermann, M. (1993) Fields of values and iterative methods. Linear Algebra Appl., 180, 167-197.

Eiermann, M. \& ERnst, O. G. (2001) Geometric aspects in the theory of Krylov subspace methods. Acta Numer., 10, 251-312.

Eisenstat, S. C., Elman, H. C. \& Schultz, M. H. (1983) Variational iterative methods for nonsymmetric systems of linear equations. SIAM J. Numer. Anal., 20, 345-357.

Elman, H. C., Silvester, D. J. \& Wathen, A. J. (2005) Finite Elements and Fast Iterative Solvers: with applications in incompressible fluid dynamics. Oxford, UK: Oxford University Press.

Golub, G. H. \& VAn LoAn, C. F. (1996) Matrix Computations, third edn. Baltimore, MD: The John Hopkins University Press.

Greenbaum, A., Pták, V. \& Strakoš, Z. (1996) Any nonincreasing convergence curve is possible for GMRES. SIAM J. Matrix Anal. Appl., 17, 465-469.

Greenbaum, A. (1997) Iterative Methods for Solving Linear Systems. Philadelphia, PA: SIAM.

Greenbaum, A. \& Strakoš, Z. (1994) Matrices that generate the same Krylov residual spaces. Recent Advances in Iterative Methods (G. Golub, A. Greenbaum \& M. Luskin eds). IMA Volumes in Mathematics and its Applications, vol. 60. New York, NY: Springer.

Greenbaum, A. \& Trefethen, L. N. (1994) GMRES/CR and Arnoldi/Lanczos as matrix approximation problems. SIAM J. Sci. Comput., 15, 359-368.

HemmingSSON, L. (1998) A semi-circulant preconditioner for the convection-diffusion equation. Numer. Math., 81, 211-248.

Higham, N. J. (2002) Accuracy and Stability of Numerical Algorithms, second edn. Philadelphia, PA: SIAM.

Horn, R. A. \& Johnson, C. R. (1999) Topics in Matrix Analysis. Cambridge, UK: Cambridge University Press. 
HuhtAnEn, M. (1999) Pole assignment problems for error bounds for GMRES. Technical Report A418. Helsinki, Finland: Helsinki Univeristy of Tehhnology Institute of Mathematics.

Huhtanen, M. \& Nevanlinna, O. (2000) Minimal decompositions and iterative methods. Numer. Math., 86, 257-281.

IPSEN, I. C. F. (2000) Expressions and bounds for the GMRES residual. BIT, 40, 524-535.

JENNINGS, A. (1977) Influence of the eigenvalue spectrum on the convergence rate of the conjugate gradient method. J. Inst. Math. Appl., 20, 61-72.

LI, R.-C. \& ZHANG, W. (2009) The rate of convergence of GMRES on a tridiagonal Toeplitz linear system. Numer. Math., 112, 267-293.

LiESEn, J. (2000) Computable convergence bounds for GMRES. SIAM J. Matrix Anal. Appl., 21, 882-903.

Liesen, J. \& Strakoš, Z. (2004) Convergence of GMRES for tridiagonal Toeplitz matrices. SIAM J. Matrix Anal. Appl., 26, 233-251.

LIESEN, J. \& STRAKOŠ, Z. (2005) GMRES convergence analysis for a convection-diffusion model problem. SIAM J. Sci. Comput., 26, 1989-2009.

Liesen, J. \& StRAKoš, Z. (2010) On the computational cost of Krylov subspace methods for solving linear algebraic systems. Preprint.

LiESEN, J. \& TICHÝ, P. (2012) The field of values bound on ideal GMRES. Technical Report. T. U. Berlin.

Meijerink, J. A. \& VAN Der Vorst, H. A. (1977) An iterative solution method for linear systems of which the coefficient matrix is a symmetric $M$-matrix. Math. Comp., 31, 148-162.

Nevanlinna, O. (1996) Convergence of Krylov methods for sums of two operators. BIT, 36, 775-785.

Ramage, A. (1999) A multigrid preconditioner for stabilised discretisations of advection-diffusion problems. $J$. Comput. Appl. Math., 110, 187-203.

SAAD, Y. (1996) Iterative Methods for Sparse Linear Systems. Philadelphia, PA: SIAM.

SAAD, Y. \& SCHULTZ, M. H. (1986) GMRES: a generalized minimal residual algorithm for solving nonsymmetric linear systems. SIAM J. Sci. Stat. Comput., 7, 856-869.

Silvester, D., Elman, H. \& Ramage, A. (2011). http://www.manchester.ac.uk/ifiss/.

Simoncini, V. \& SZYLD, D. B. (2007) Recent computational developments in Krylov subspace methods for linear systems. Numer. Linear Algebra Appl., 14, 1-59.

ToH, K.-C. (1997) GMRES vs. ideal GMRES. SIAM J. Matrix Anal. Appl., 18, 30-36.

ToH, K.-C., TodD, M. J. \& TÜTÜNCÜ, R. H. (1999) SDPT3-a Matlab software package for semidefinite programming. Optim. Method. Softw., 11, 545-581.

Toh, K.-C. \& Trefethen, L. N. (1998) The Chebyshev polynomials of a matrix. SIAM J. Matrix Anal. Appl., 20, 400-419.

Trefethen, L. N. (1990) Approximation theory and numerical linear algebra. Algorithms for Approximation II (J. C. Mason \& M. G. Cox eds). London, UK: Chapman and Hall.

VAN DER SluIS, A. (1969) Condition numbers and equilibration of matrices. Numer. Math., 14, 14-23.

VAN DER Vorst, H. A. \& VUIK, C. (1993) The superlinear convergence behaviour of GMRES. J. Comput. Appl. Math., 48, 327-341. 